Orange Journal/Volumen 1 Número 1/ Enero - Junio 2019

DOI: http://dx.doi.org/issn.2710-995X/2019.1.03

\title{
Requisitos previos para la formación de paradigmas de gestión modernos en términos de evolución del conocimiento científico.
}

Requisitos prévios para a formação de paradigmas de gestão avançada em termos de evolução do conhecimento científico.

Recibido: 17 de marzo de $2019 \quad$ Aceptado: 24 de junio de 2019

Escrito por:

Elena V. Krasova ${ }^{7}$

Aleksey V. Osipov ${ }^{8}$

Evgeniy V. Hilko ${ }^{9}$

\section{Resumen}

La investigación sobre los temas relevantes para la comprensión de las direcciones en que se está moviendo el conocimiento científico en economía y gestión es un componente importante de la ciencia moderna. Este conocimiento se forma y resume a través de paradigmas científicos. El enorme impacto que tiene la gestión en todo tipo de actividades económicas determina la importancia de los paradigmas de gestión al seleccionar la forma más efectiva en el desarrollo socioeconómico. El propósito de la investigación es examinar los requisitos previos para la aparición de tales paradigmas en términos de evolución del conocimiento científico en economía y gestión. El artículo propone la definición del paradigma de gestión del autor como categoría científica y examina los factores científicos e históricos detrás de la formación del paradigma de gestión clásico y revela un vínculo orgánico entre los paradigmas de gestión y los de desarrollo socioeconómico. En particular, el artículo analiza el proceso de transición gradual del paradigma clásico de gestión al innovador. El autor estudia y compara los enfoques adoptados por los principales científicos en este campo y determina los modelos científicos dominantes formados dentro del paradigma de la innovación. El tema de estudio de tales modelos y categorías cruciales de la ciencia moderna, es decir, gestión de la innovación, economía del conocimiento, capital intelectual, capital organizacional, capital humano y una serie de otras categorías.

\footnotetext{
${ }^{7}$ Candidate of Economic Sciences (PhD in Economics) Vladivostok State University of Economics and Service, Associate Professor at the Department of Economics \& Management, Vladivostok, Russia.

8 Assistant of the Department of Tourism and Ecology Vladivostok State University of Economics and Service, Vladivostok, Russia.

${ }^{9}$ Undergraduate of the educational program International Economy, Vladivostok State University of Economics and Service, 41 Gogol Street, Vladivostok, Russia.
} 


\section{ORANGE JOURNAL}

Palabras clave: Paradigma de gestión, evolución de la teoría de gestión, evolución de la teoría económica, paradigma de gestión clásico, paradigma de gestión de la innovación, economía del conocimiento, capital intelectual.

\section{Resumo}

A investigação sobre os temas relevantes para a compreensão das direcções que estão a ser deslocados no âmbito dos estudos científicos sobre economia e gestão é um componente importante da ciência moderna. Este conocimiento se forma e resume os paradigmas científicos. O enorme impacto que tem a gestão em todo o tipo de atividades determinantes determinam a importância dos paradigmas de gestão para a seleção mais efetiva no desenvolvimento socioeconômico. O objetivo da investigação é a análise das necessidades do aluno para a análise dos paradigmas em termos de evolução do desenvolvimento dos estudos científicos em economia e gestão. $\mathrm{O}$ artigo propôs a definição do paradigma da gestão como a análise científica e a análise dos fatores científicos e os resultados negativos da formação da paradigma da gestão clássica e revela um resultado orgânico entre os paradigmas da gestão e do desenvolvimento socioeconómico. Em particular, o artigo intitula o processo de transição gradual do paradigma clássico de gestión al innovador. O autor estuda e compara os enfoques adotados pelos principais científicos neste campo e determina os modelos científicos dominantes formados dentro do paradigma da inovação. $\mathrm{O}$ tema de estudo de séries e matérias-primas da ciência moderna, a decisão, a gestão da inovação, a economia do capital, o capital intelectual, o capital organizacional, o capital humano e uma série de outras categorias.

Palavras-chave: Paradigma de gestão, evolução da teoria da gestão, evolução da teoria econômica, paradigma da gestão clássica, paradigma da gestão da inovação, economia do conhecimento, capital intelectual.

\section{Introducción}

La ciencia moderna estudia las direcciones en el desarrollo socioeconómico de los países e incluso los grupos de países sobre la base de los cambios en los paradigmas de desarrollo económico, ya que una política socioeconómica en los países desarrollados se basa en las disposiciones de las teorías generales. Como resultado de un desarrollo histórico complicado a largo plazo, los paradigmas se forman al combinar todas las características útiles de la experiencia pasada con nuevos conocimientos basados en el análisis de procesos y fenómenos modernos (Kuzheva, 2015). Un paradigma, que sigue siendo poco estudiado, pero tiene un gran potencial para la formación de etapas de desarrollo de las economías nacionales, es un paradigma de gestión.

En términos de su significado filosófico, asignado al paradigma por Thomas Kuhn, un filósofo e historiador estadounidense de la ciencia (Kuhn, 1969), un paradigma de gestión podría ser imaginado como un conjunto de teorías, técnicas, valores y problemas de gestión, que comparten las élites científicas y de gestión de un país en una era particular. El paradigma de gestión es un fenómeno metodológico, una ideología y un conjunto de patrones de comportamiento, tecnologías y métodos utilizados para gestionar los sistemas 
sociales (Tsvetkov, 2016). Utilizando esta definición y las opiniones de otros científicos líderes (Abalkin, 2001; Glazyev, 2016), los autores consideran un paradigma de gestión como una o más teorías y conceptos de gestión dominantes, que son universalmente reconocidos en una situación económica dada durante un período determinado y desarrollo social directo en general o dentro de un país en particular. Al combinarse y ajustarse a los detalles históricos, culturales y políticos de un país, estas teorías de gestión coexisten, interactúan, se enriquecen mutuamente, se enfrentan y actúan como "polos de tensión" en el campo de gestión, creando así varias síntesis y formas mixtas y de transición (Slonov, 2005).

El concepto de paradigma de gestión aún no ha obtenido su significado común en la ciencia moderna. Sin embargo, es obvio que un cambio en estos paradigmas constituye un fenómeno tan objetivo como un cambio en las élites gobernantes, ya que determina los requisitos previos y las condiciones para las transformaciones de la sociedad socioeconómica.

En la actualidad hay dos paradigmas paralelos de gestión moderna, los convencionales y los de innovación. El propósito de la investigación es examinar los requisitos previos para la ocurrencia de tales paradigmas en términos de evolución del conocimiento científico en economía y gestión. La importancia de la investigación se debe a la necesidad de comprender las expresiones de varios paradigmas, especialmente en el entorno de la formación de la economía postindustrial, que corresponde al paradigma de gestión de la innovación.

\section{Métodología}

La base teórica y metodológica del artículo son las disposiciones generales de la ciencia económica y administrativa moderna, en particular: teoría neoclásica moderna, teoría del desarrollo de la economía mundial, concepto de organización científica laboral, teoría de la génesis del capitalismo, concepto de desarrollo innovador, aplicado a través del análisis del sistema. En el marco de estas teorías, se consideran los enfoques más significativos para la ciencia de la gestión en innovaciones, conocimiento y capital intelectual, que formaron el paradigma moderno de gestión de la innovación.

La investigación se basa en los métodos de análisis del sistema, estudios comparativos, abstracción científica y enfoque historiográfico en unidad con el enfoque dinámico.

El uso del enfoque del sistema permite considerar los detalles del objeto de investigación. La investigación se basa en el aparato conceptual clásico, desarrollado por la ciencia mundial, que permite estudiar las condiciones de formación de varios paradigmas de gestión desde el punto de vista de la evolución del conocimiento científico.

\section{Resultados y discusión}

Requisitos previos para la formación del paradigma clásico de gestión. 


\section{ORANGE JOURNAL}

Las fuentes de los paradigmas de gestión como teoría independiente se remontan a la era del desarrollo del modo de producción capitalista en los siglos XIX y los intentos de explicar el crecimiento de la productividad como el principal signo en la génesis del capitalismo. Karl Marx (1818-1883), el científico alemán que dejó un extenso patrimonio científico en el campo de la gestión de empresas capitalistas, consideró indispensable el vínculo entre la propiedad y la gestión del capitalismo, ya que combinó el proceso de gestión de la producción y el proceso de crecimiento del capital. "La razón por la que un capitalista es capitalista no es porque administra una empresa industrial. Todo lo contrario, se convierte en gerente de la industria porque es capitalista" (Marx, 2017, p. 311). Mientras un capitalista busque las formas de mejorar la eficiencia del capital, es un emprendedor como tal. Luego, un crecimiento de capital exitoso siempre va acompañado de los procesos de diferenciación de la actividad administrativa y la transición de la autoridad a un nivel jerárquico inferior. "Al igual que un ejército que necesita a sus oficiales y sargentos, los trabajadores unidos por un trabajo común bajo el mando de la misma capital necesitan gerentes industriales y personas que pasan por alto, quienes, mientras se realiza el trabajo, comandan en nombre de la capital" (Marx, 2017, p. 310). Por lo tanto, en la producción a gran escala, los gerentes contratados, no los capitalistas, se convierten en conductores de la actividad capitalista y, en consecuencia, del desarrollo empresarial. Considerar el papel de los gerentes como personas que pasaron por alto llevó a Marx a pensar en la inevitabilidad de los conflictos de clase, la autodestrucción del capitalismo y, como resultado, la conveniencia de transferir las funciones de gestión de la industria de individuos particulares a la sociedad, que gestionará la producción en interés público, en de conformidad con el plan público y con todos sus miembros involucrados (Osipov y Embulaev, 2012).

Frederick W. Taylor (1856-1915), un ingeniero y científico estadounidense, señaló el papel que desempeña la organización científica laboral y de gestión en el desarrollo económico y tomó el sistema de gestión científica como base para el progreso económico, social y técnico en la sociedad (Taylor, 1991). El enfoque de Taylor condujo objetivamente a la separación de los términos "propietario" y "gerente" al justificar que la producción debe ser administrada por personas adecuadas y apropiadas, es decir, gerentes profesionales.

Contrariamente al postulado de Marx sobre la inevitabilidad de los conflictos de clase y la autodestrucción del capitalismo, la teoría de Taylor permitió la posibilidad principal y la posibilidad de establecer relaciones armónicas (e incluso de socios) entre propietarios de empresas y trabajadores contratados debido a su interés mutuo en el crecimiento de la productividad laboral. basado en la intensificación de los procesos de capacitación y la acumulación de conocimiento (Sorochaikin, 2011). Así, el taylorismo proporcionó la base para el primer paradigma clásico, estable de gestión.

Paradigmas de gestión y paradigmas de desarrollo socioeconómico en los siglos XX y XXI

El surgimiento contemporáneo del marxismo y el taylorismo, es decir, dividido en las opiniones del papel de los propietarios en la gestión, predeterminó las bases ideológicas para la justificación del socialismo, por un lado, y un mayor desarrollo del capitalismo, 
por otro lado. Formó puntos de vista esencialmente diferentes de las relaciones de propiedad y el papel del estado en la economía y también determinó paradigmas de gestión económica estatal dramáticamente diferentes. Sin embargo, en términos de gestión de la organización, el paradigma clásico de gestión, que identificó el crecimiento de la productividad laboral como el principal propósito de la gestión, resultó ser muy resistente tanto para los modos de producción capitalista como socialista. Es cierto que las metodologías de Taylorismo que proporcionan la estructuración y la estandarización de los flujos de trabajo, el control estricto sobre su ejecución y el sistema de recompensa / penalización parecían garantizar una solución a cualquier problema de gestión (Tsvetkov, 2016).

Amplios estudios sobre cuestiones organizativas y de gestión realizadas en el siglo XX transformaron la gestión en un campo crucial de la ciencia global y le dieron una gran importancia social y económica. El paradigma clásico de gestión era interdependiente y se fusionó orgánicamente con la teoría económica neoclásica, que se formó a fines del siglo XIX como afectada por los puntos de vista del laissez faire y sigue siendo dominante en la actualidad. Los paradigmas económicos clásicos correspondían perfectamente a la era del capitalismo, y en el mundo moderno, liberan y regulan una economía marcada debido a su simplicidad y sus prerrequisitos aparentemente obvios, siendo los principales una racionalidad excepcional de elección económica, recursos limitados y la necesidad de alcanzar el equilibrio sobre la base del mecanismo de mercado (Cowan, 2004). Poder metodológico y transparencia del paradigma neoclásico.

Las herramientas matemáticas lógicamente perfectas lo hicieron muy resistente y fácil de usar por numerosos científicos durante el análisis de procesos y fenómenos económicos particulares. Sin embargo, hoy en día algunos expertos sostienen que este proceso se ha convertido en una cita infinita de los detalles en sistemas socioeconómicos complejos sin un éxito visible, mientras que se requiere el desarrollo de nuevos modelos de existencia social (Golovanov, 2012). La teoría neoclásica y sus derivados están sujetos a críticas por parte de científicos extranjeros y nacionales, que esencialmente exigen una nueva corriente principal en la teoría económica (Blaug, 2004; Yerznkyan, 2004; Hodgson, 2008; Kirdina, 2013). Como respuesta al paradigma neoclásico que ya no satisfizo las necesidades del progreso económico y social en la segunda mitad del siglo XX, surgieron una serie de teorías que competían con la teoría neoclásica o que la complementaban y ampliaban sustancialmente. Los más importantes incluyen la teoría de la sociedad postindustrial, el paradigma de la evolución, el paradigma institucional y de la evolución, el paradigma de la innovación y el paradigma multicultural (Slonov, 2005; Hodgson, 2008; Moroz, 2011; Vojcehovskij, 2016; Tsvetkov, 2016).

Cada una de las teorías anteriores presenta sus propios factores para el cambio en la trayectoria del desarrollo económico y cambia el énfasis de la gestión económica en su propia forma específica. Los enfoques de gestión alternativos se basan principalmente en el conocimiento, las cualidades personales y profesionales de los gerentes, el factor humano y, finalmente, los recursos humanos. Por lo tanto, se forma el paradigma de gestión de la innovación, con sus diversos componentes sometidos a un examen exhaustivo como los más correspondientes al tipo de desarrollo económico postindustrial (Lazarev y Krasova, 2018). 


\section{ORANGE JOURNAL}

Paradigma de gestión de la innovación: naturaleza específica de los enfoques científicos.

Según la sabiduría convencional, la base del paradigma de la innovación fue establecida por Joseph A. Schumpeter (1883-1950), el conocido economista austriaco. Su concepto combina la justificación económica de la función empresarial de la administración con la presentación del retrato psicológico del empresario. Schumpeter consideró a un emprendedor como el factor principal detrás del desarrollo del capitalismo y describió el emprendimiento como un proceso de "destrucción creativa". En su libro "Teoría de la economía Desarrollo "un empresario se define como un innovador, un jugador clave en el sistema de gestión. Sus funciones son ejecutar innovaciones, que juegan un papel importante en el desarrollo de la economía capitalista, y asegurar el crecimiento económico (Schumpeter, 2008; Osipov, 2017).

Según las opiniones de Peter Drucker (1909-2005), un científico estadounidense, la actividad de innovación de los gerentes proporciona la base para el desarrollo de la sociedad. Un gerente, que ha dominado los métodos de la actividad de innovación, se convierte en gerente-empresario llevando a cabo una "revolución de gestión". En la sociedad Drucker, el factor principal detrás del crecimiento de la productividad laboral es una gestión adecuada y científicamente justificada, es decir, una gestión basada en el conocimiento estricto de las regularidades y la dinámica del proceso de producción: el conocimiento aplicado a la organización laboral asegura un crecimiento explosivo de su productividad (Drucker, 2006).

John K. Galbraith (1908-2006), un científico estadounidense, estableció el proceso de surgimiento en la tecno estructura corporativa como un límite para el surgimiento del "nuevo estado industrial". La tecno estructura corporativa es un conjunto de una gran cantidad de científicos, ingenieros y técnicos; expertos en distribución, publicidad y ventas; expertos en relaciones públicas, cabilderos, abogados y otros profesionales. La tecno estructura desplazó los procesos de toma de decisiones de los propietarios de capital al monopolizar el conocimiento requerido para la toma de decisiones y son los gerentes quienes controlan la tecno estructura basada en tecnologías modernas y métodos de planificación. Galbraith se convirtió en un ideólogo universalmente reconocido de la reforma liberal y justificó el concepto de transformación del capitalismo en sus libros "The New Industrial State" (1967) y "Economics and the Public Purpose" (1973) (Galbraith, 1976. P. 155).

Los incuestionables logros de Galbraith incluyen, entre otras cosas, la introducción del término "capital intelectual" en el vocabulario científico de la gerencia (1969). Sin embargo, Thomas Stewart, un publicista y economista estadounidense, comenzó un estudio más detallado de esta categoría en 1991. El libro "Capital intelectual" de Stewart, publicado en 1997, describe los problemas cruciales de la economía, donde el conocimiento y la información son los principales recursos de producción. El científico define el capital intelectual como un conjunto de patentes, procesos, habilidades de gestión, tecnologías, experiencia y datos sobre consumidores y proveedores.

Con respecto a la gestión empresarial, el científico aclaró que el capital intelectual era la cantidad acumulada de conocimiento que posee todo el personal de la empresa, lo que 
garantiza su competitividad, es decir, el conocimiento que obtiene su forma tangible (Stewart, 2007).

Además de Galbraith y Stewart, varios científicos de diferentes países estudiaron los temas relevantes para la economía del conocimiento y la gestión del capital intelectual, y la mayoría de ellos compartieron puntos de vista similares sobre este tema. Por ejemplo, L. Edvinsson define una categoría de capital intelectual como conocimiento que puede convertirse en valor (Edvinsson, 2000). En el artículo "Activos intangibles", J. Daum escribió que el capital intelectual era conocimiento y habilidades estructurados basados en los vínculos y el potencial de desarrollo y creación de valor (Daum, 2002).

Los científicos modernos normalmente identifican tres elementos principales dentro de la estructura del capital intelectual: capital humano, capital de relación (capital del consumidor y del cliente) y capital estructural (organizacional). Estos elementos pueden tener varias interrelaciones entre sí. Por ejemplo, en el modelo del "Esquema de valor de Scandia", el capital humano incluye las competencias y habilidades del personal de la empresa (Edvinsson, 2000). El capital estructural incluye los elementos acumulados por la empresa como resultado del desempeño de gerentes y empleados. El capital estructural se divide en capital del cliente, es decir, el valor presentado por las relaciones con los clientes y el organizacional. En este modelo, el capital organizacional comprende capital de innovación (patentes, acuerdos de licencia, etc.) de la imagen y la reputación, que determinan en gran medida el valor de la empresa (marcas registradas, marcas) y el capital de proceso (infraestructura de la empresa, tecnologías de la información, software, flujos de trabajo etc.)

K.E. da otra estructura de capital intelectual ampliamente conocida. Sveiby en su libro "El monitor de activos intangibles". Sveiby usó el término "activos intangibles" en el sentido del capital intelectual. En esta clasificación, la categoría de capital intelectual se divide en parte externa, parte interna y competencias del personal de la entidad. La estructura externa es un capital de consumo que incluye relaciones positivas con consumidores, proveedores, competidores y organismos gubernamentales. La estructura interna es un capital organizativo, en particular patentes, derechos de autor, software, diseños industriales, bases de datos, esquemas de cultura corporativa y otros elementos de propiedad intelectual. Las competencias del personal son capital humano que incluye el nivel educativo, experiencia profesional y competencias, habilidades comunicativas, nivel ético general, etc. (Sveiby, 1997).

El modelo descrito en "FiMIAM: método financiero de medición de activos intangibles" por I. Rodov y F. Leliaert también divide el capital intelectual en tres partes: capital humano, capital del cliente y capital estructural. Cada una de estas partes incluye una serie de elementos de capital intelectual. En particular, las competencias profesionales, la reputación, la experiencia, las capacidades y las habilidades se identifican en el capital humano. Al mismo tiempo, algunas categorías resultan estar en el llamado cruce de los términos: por ejemplo, la lealtad de los clientes se refiere tanto al capital humano como al capital del cliente; la marca registrada y la marca pertenecen tanto al capital estructural como al capital del cliente; Los conocimientos pertenecen simultáneamente a tres componentes del capital intelectual (Rodov y Leliaert, 2002). 
Por lo tanto, cada elemento del capital intelectual tiene su propósito y funciones. En numerosos modelos de gestión, el capital organizativo o estructural significa las oportunidades organizativas de la empresa para responder a los desafíos modernos del mercado mediante el uso y la transformación de datos. El capital organizacional es propiedad de la compañía en gran medida y puede ser un sujeto de intercambio y adquisición de capital adicional relativamente independiente. El capital del consumidor o cliente significa enlaces y comunicaciones estables con las contrapartes, información sobre las contrapartes y la política del cliente (Shashlo, Petruk y Korostelev, 2018).

En cuanto a la teoría del capital humano, es una sección relativamente reciente en la teoría económica moderna y el paradigma de gestión. Recorrió un largo camino desde la definición de sus términos fundamentales hasta el desarrollo y la aprobación de métodos de gestión integrales en varios niveles con el fin de un uso efectivo y la ejecución del potencial humano.

\section{Conclusiones}

Por lo tanto, incluso hoy en día, un ser humano educado, desarrollado profesionalmente y creativo, que está personalmente interesado en la prosperidad de su entidad y sociedad, es considerado como el principal recurso del desarrollo económico (Slonov, 2005). Como sujeto de control de gestión, una entidad con fines de lucro o sistema económico que buscaba el equilibrio fueron reemplazados por conocimiento (información). En estas condiciones, la gerencia proporciona base metodológica para una gestión eficaz de la información dirigida a lograr los resultados (productos, tecnologías, etc.) que serán los mejores para la sociedad. Específicamente, la gestión es un conjunto de operaciones para la racionalización racional, la organización y la sistematización de la información (conocimiento) con el fin de establecer la forma más efectiva de aplicarlo en interés público.

A pesar de la obvia conveniencia de alejarse del paradigma clásico de gestión debido a la acumulación de una masa crítica de las tareas que no se pueden resolver dentro de su marco, todavía no se ha creado una teoría alternativa unificada. La idea principal que reemplaza el concepto de gestión convencional, es decir, el paradigma de gestión de la innovación, con sus signos y características, está sujeto a los estudios anteriores y a muchos otros, actualmente existe como una estructura teórica y se implementa por partes (Tsvetkov, 2016). Al mismo tiempo, el paradigma de la innovación muestra claramente el desarrollo futuro de la dirección de gestión y actúa como una base para desarrollar los métodos y herramientas de la corriente principal de gestión más reciente.

\section{Referencias}

Abalkin, L.I. (2001) En busca de un nuevo paradigma de desarrollo social y económico. Ciencia y poder: memorias de humanistas y científicos sociales. Moscú, Science Publ. Blaug, M. (2004). La metodología de la ciencia económica, o cómo explican los economistas. Moscú, Issues of Economics Publ.

Cowan, R. (2004). Dependencia del camino, causalidad y política económica. El Heraldo Económico de la Universidad Estatal de Rostov, vol. 2 (4). Páginas. 10-29. 
Daum, J.H. (2002) Más allá del presupuesto: ¿un modelo para la gestión y el control del desempeño en el siglo XXI? Boletín alemán "Controlling \& Finance", julio. Disponible en línea en: https://www.coursehero.com/file/18166055/Da um-2002 /.

Drucker, P.F. (2006) The Essential Drucker (Enciclopedia de la gestión). Moscú, Williams Publ.

Edvinsson, L. (2000). Algunas perspectivas sobre intangibles y capital intelectual 2000. Journal of Intellectual Capital, vol. 1. págs. 12-16.

Galbraith, J.K. (1976) La economía y el fin público. Moscú, Progress Publ.

Glazyev, S.Yu. (2016) Un nuevo paradigma de la ciencia económica. Gestión del gobierno,

Vol. 56. págs. 5-39.

Golovanov, A.I. (2012) Metodología de una ciencia económica moderna: definición del vector general. El Heraldo de la Universidad Estatal de Tomsk. Economía, vol. 3. Pp. 510 .

Hodgson, G. (2008). ¿La economía evolutiva e institucional como la nueva corriente principal? El Heraldo Económico de la Universidad Estatal de Rostov, vol. 6 (2). pp. 8 21.

Kirdina, S.G. (2013). Individualismo metodológico e institucionalismo metodológico. Issues of Economics, vol. 10. págs. 66-89.

Kuhn, T.S. (1969) La estructura de las revoluciones científicas. Moscú, Progress Publ.

Kuzheva, S.N. (2015) De la historia de las innovaciones y teorías de la innovación. Innovative Economics and Society, vol. 2. págs. 2-12.

Lazarev, G.I., Krasova, E.V. (2018) Investigación y desarrollo en China: alcance y características específicas del proceso de innovación. Amazonia Investiga, vol. 7 (14). pp. 73-83.

Marx, K. (2017). Capital. Volumen 1. Moscú, Capital Series Publ.

Moroz, V.N. (2011) Paradigmas de la ciencia económica y posibilidad de su aplicación en la gestión innovadora. El Gerald del Instituto de Derecho de Kaliningrado del Ministerio del Interior de Rusia, vol. 1. Pp. 100-104.

Osipov V.A. (2017) Ciclicidad de paradigmas de gestión en la economía rusa. Direcciones prioritarias para el desarrollo de la economía del Lejano Oriente. Actas de la Conferencia Regional Científico-Práctica. Vladivostok: FEFU. Páginas. 150-161.

Osipov, V.A., Embulaev, V.N. (2012) El beneficio como objetivo de la toma de decisiones de gestión en el ámbito del emprendimiento. Revista científica en línea "Naukovedeniye", Vol. 4 (13). P. 48. Disponible en línea en:

https://naukovedenie.ru/PDF/93evn412.pdf. Rodov, I., Leliaert, Ph. (2002). FiMIAM: método financiero de medición de activos intangibles. Revista de Capital Intelectual, vol. 3. págs. 323- 336.

Schumpeter, J.A. (2008) Teoría del desarrollo económico. Moscú, Direct Media Publ. Shashlo, N.V., Petruk, G.V. Y Korostelev,

AUTOMÓVIL CLUB BRITÁNICO. (2018) Determinantes de la interacción de integración entre los sujetos del ecosistema de innovación empresarial de la macro región. Amazonia Investiga, vol. 7 (13). pp. 351-363.

Slonov, N.N. (2005) Paradigmas de gestión. The Gerald of the Volga Academy of Government Service, vol. 8. págs. 50-58. 


\section{ORANGE JOURNAL}

Sorochaikin, A.N., Sorochaikin, I.A. (2011) Conocimiento y crecimiento de la productividad del trabajo (concepción de P. Drucker - F. Taylor). El Boletín de la Universidad Estatal de Samara, vol. 10. págs. 15-21.

Stewart, T.A. (2007) Capital intelectual: la nueva riqueza de las organizaciones. Nueva York, Doubleday Publ. 278 p.

Sveiby, K.E. (1997) La nueva riqueza organizacional. San Francisco: Berrett-Koehler. Taylor, F.W. (1991). Los principios de la gestión científica. Moscú: Controlling Publ.

Tsvetkov, A.N. (2016) Paradigma de la gestión moderna: demostración en las organizaciones. Estrategias de negocios, vol. 6. págs. 30-34.

Vojcehovskij, S.N. (2016) Relación de paradigma de equilibrio, paradigma evolutivo y paradigma sistémico en el desarrollo de la teoría económica. El Heraldo de la Ciencia Moderna, vol. 2-1. pp. 60-63.

Yerznkyan, B.A. (2004) Schumpeter, la corriente principal y la teoría evolutiva del desarrollo económico. Ciencia económica de la Rusia moderna, vol. 4. pp. 53-67. 